\title{
ENXERTOS ÓSSEOS ALVEOLARES NA FISSURA LABIOPALATINA: PROTOCOLOS ATUAIS E PERSPECTIVAS FUTURAS
}

\section{ALVEOLAR BONE GRAFT IN CLEFT LIP AND PALATE: CURRENT PROTOCOLS AND FUTURE PROSPECTS}

\author{
Erica Alexandra Macedo Pessoa* \\ Andre Braune* \\ Priscila Ladeira Casado*** \\ Patricia Nivoloni Tannure ${ }^{* * *}$
}

\begin{abstract}
RESUMO
A fissura labiopalatina é a malformação congênita mais comum do ser humano, envolvendo a face e a cavidade bucal. Uma das fases do tratamento reabilitador consiste, na maioria dos casos, na movimentação dentária no local onde há ausência de suporte ósseo. Isso só é possível com a realização do enxerto ósseo alveolar na região da fissura, objetivando-se o suporte e reconstrução do arco dentário e o fechamento de fístula nasal. O enxerto realizado na época de dentição mista, antes da irrupção do canino permanente, é considerado um procedimento fundamental e quando retirado da crista ilíaca do próprio paciente tem as vantagens de um material autógeno cuja incorporação é favorecida. Entretanto, pesquisas têm sugerido o uso de proteína morfogenética óssea recombinante humana para enxertia em pacientes fissurados. O objetivo deste artigo é revisar a literatura atual sobre enxertos ósseos alveolares e discorrer sobre as perspectivas futuras diante de novos materiais disponíveis.
\end{abstract}

Descritores: Fenda labial • Fissura palatina • Transplante ósseo • Reabilitação bucal.

\section{ABSTRACT}

Cleft lip and palate is the most common congenital malformation of the human involving the face and oral cavity. A rehabilitation stage of treatment consists, in most cases, the tooth movement in the location where there is no bone support. This is only possible with the completion of alveolar bone grafting in the cleft region, aiming to support and reconstruction of the arch and the nasal fistula closure. Bone graft performed in mixed dentition prior to permanent canine eruption is considered a key procedure and when taken from the iliac crest of the patient has the advantages of autogenously material whose incorporation is favored. However, research has suggested the use of recombinant human bone morphogenetic protein for grafting onto cleft. The aim of this article is to review the current literature on alveolar bone grafts and discuss future prospects ahead to new materials available.

Descriptors: Cleft lip $\bullet$ Cleft palate $\bullet$ Bone transplantation $\bullet$ Mouth rehabilitation

\footnotetext{
* Mestranda em Odontologia (Reabilitação bucal) da Universidade Veiga de Almeida (UVA), Rio de Janeiro (RJ)

** Chefe substituto do Centro de Cirurgia Crânio-Maxilofacial do Instituto Nacional de Traumatologia e Ortopedia (INTO), Rio de Janeiro (RJ)

*** Doutora em Morfologia e Professora Adjunta do Departamento de Periodontia da Universidade Federal Fluminense (UFF), Niterói (RJ).

**** Doutora em Odontologia e Docente do Mestrado Profissional em Odontologia da Universidade Veiga de Almeida (UVA), Rio de Janeiro (RJ)
} 
PESSOA EAM

BRAUNE A

CASADO PL

TANNURE PN

ENXERTOS ÓSSEOS

ALVEOLARES

NA FISSURA

$\angle A B I O P A L A T I N A$ :

PROTOCOLOS

ATUAIS E

PERSPECTIVAS

FUTURAS

REV, ODONTOL, UNIV, CID, SÃO

PAULO

$2015 ; 27(1): 49-$

55, JAN-ABR

\section{N T R O D U ÇÃ O}

O desenvolvimento craniofacial é extremamente complexo por envolver diversas estruturas anatômicas e processos moleculares. Alterações durante esse desenvolvimento podem resultar em diversas anomalias. Dentre as anomalias craniofaciais, a fissura labiopalatina destaca-se por ser a malformação congênita mais comum do ser humano, envolvendo a face e a cavidade bucal e atinge em torno de 1,7 em 1000 nascidos vivos com variações dependendo da etnia e região geográfica ${ }^{1}$. No Brasil, em 2011, estimou-se o nascimento de 2900 a 4400 crianças portadoras de fissuras orais ${ }^{2}$, causadas por falhas na fusão entre os processos maxilares e os processos palatinos durante a embriogênese ${ }^{3}$, podendo levar a alterações na fala, audição, estética e impacto emocional com consequências negativas na saúde, na integração social do indivíduo e na sua qualidade de vida ${ }^{1,4}$.

Pacientes portadores de fissuras orais são submetidos a tratamentos multidisciplinares complexos e de longo prazo. A movimentação ortodôntica é indispensável na maioria dos casos, entretanto, o defeito ósseo alveolar presente em função das fissuras que envolvem o rebordo alveolar limita a possibilidade de movimentação dentária nessa região, tornando o enxerto ósseo um procedimento fundamental na época da dentadura mista.

As indicações dos enxertos ósseos alveolares (EOA) estão relacionadas à necessidade de osso de suporte para os dentes erupcionados ou não adjacentes à fissura, na estabilização da pré-maxila, nos casos de fissuras bilaterais, na continuidade do rebordo alveolar, no suporte da base alar e do contorno nasolabial e na eliminação da fístula oronasal ${ }^{5,6,7}$. Os enxertos autógenos são considerados "padrão ouro" para as cirurgias de enxerto, pois é o único material que possui propriedades osteogênicas, reunindo todos os princípios biológicos para adequada incorporação ${ }^{8}$. Entretanto, alguns fatores têm levado à busca de novas soluções para substituir o osso da crista ilíaca ${ }^{9}$, como, por exemplo, o alto custo das cirurgias e as complicações pós-operatórias ${ }^{10}$.
Assim, em função da importância de se conhecerem as características dos materiais que compõem os enxertos ósseos e de se utilizar aquele com o melhor custo benefício, o objetivo deste trabalho é revisar a literatura sobre enxertos ósseos alveolares na reabilitação de pacientes fissurados, com a finalidade de otimizar seu uso pelos profissionais e permitir que os pacientes se beneficiem de suas propriedades $^{11,12}$.

\section{REVISÃO DE LITERATURA}

\section{A fissura labiopalatina}

A etiologia das fissuras labiopalatinas é multifatorial, envolvendo fatores genéticos e ambientais. Diversos genes e loci candidatos foram associados às fissuras orais isoladas em diferentes estudos e populações.

A fissura labiopalatina (FL/P) pode ser isolada ou estar associada a síndromes (OMIN). Pequenas malformações adicionais são frequentes em pacientes portadores de fissuras não sindrômicas, entre estas, destacam-se as anomalias do desenvolvimento dentário ${ }^{13}$. Sabe-se que uma alta prevalência de agenesias, dentes supranumerários e alterações de forma nas coroas dentárias são características comuns na cavidade bucal desses indivíduos e podem ser consideradas parte do fenótipo individual ${ }^{13}$.

A identificação de fatores de risco envolvidos com a patologia, como o fumo materno, estilo de vida, dieta, suplementos minerais e multivitamínicos, drogas e medicamentos $^{1}$ contribui para a implementação de programas de prevenção de fissuras orais na saúde pública. Além disso, sabe-se que malformações congênitas e o câncer podem compartilhar fatores etiológicos comuns e a associação entre fissura não sindrômica e câncer tem sido observada em diferentes estudos ${ }^{14,15}$.

\section{0 tratamento cirúrgico e ortodôntico da fissura labiopalatina}

As cirurgias plásticas primárias, a queiloplastia e a palatoplastia, realizadas nos primeiros meses e anos de idade, respectivamente, reparam o defeito no tecido mole, lábio e palato, deixando uma fissura alveolar residual. A persistência do defeito no osso alveolar é um limite para o futuro 
tratamento ortodôntico ${ }^{16}$.

Por um outro lado, a intervenção cirúrgica pode resultar na formação de uma grave cicatriz ${ }^{17}$. Tanto a técnica utilizada para o reparo quanto as habilidades da equipe possuem um importante papel na obtenção de bons resultados ${ }^{18}$. Além disso, a idade em que é realizada a primeira intervenção e o tipo de fissura também podem influenciar a ocorrência de complicações no pós-operatório, especialmente no processo de cicatrização ${ }^{19}$. Portanto, é importante coordenar a época do enxerto ósseo alveolar da fissura com o movimento ortodôntico no local. A meIhor idade para o enxerto ósseo é durante a dentição mista, geralmente entre 5 e 12 anos de idade, antes da erupção do canino ou, nos casos viáveis, do incisivo lateral superior ${ }^{5,20}$.

Um dos pré-requisitos para o sucesso do enxerto alveolar é a perfeita técnica operatória evitando-se a tensão vestibular mucoperiosteal do retalho e buscando o recobrimento adequado de gengiva ceratinizada sobre o enxerto. O pré-operatório ortodôntico do tratamento não deverá causar um colapso do septo ósseo que circunda o dente adjacente à fissura, para que não haja laceração do tecido com o posicionamento do enxerto sobre as raízes do dente ${ }^{20}$.

\section{Enxertos ósseos alveolares (EO A)}

Tem sido utilizada a classificação baseada no estágio de desenvolvimento dental: enxerto primário durante o primeiro estágio de dentição; enxerto secundário durante o estágio misto de dentição; enxerto terciário após o completo segundo estágio de erupção ${ }^{20}$.

O enxerto alveolar primário, quando realizado, cria uma ponte óssea através do defeito congênito, isso não significa que a fístula será fechada ou que um osso adequado será formado. Já o enxerto secundário consegue fechar a fístula oro-nasal ${ }^{21}$. O enxerto secundário objetiva a restauração do rebordo alveolar em fissurados com eficácia no fechamento da fístula oro-nasal e estabilização dos segmentos maxilares, restaurando a crista alveolar, promovendo suporte ósseo para os dentes adjacentes à fissura, minimizando os distúrbios de crescimento da maxila, preparando o caminho para erupção dos dentes e posteriormente para movimentos ortodônticos e implantes ${ }^{22}$.

O enxerto ósseo na dentição permanente, após o tratamento ortodôntico corretivo, recebe o nome de enxerto tardio ou terciário. Apresenta o inconveniente de não resolver problemas de perda óssea nos dentes adjacentes à fissura e, ocasionalmente, provocar reabsorção radicular progressiva no terço cervical dos dentes adjacentes à fissura, principalmente o canino. Quando o enxerto é realizado na época propícia, tem ocorrência desprezível, em torno de $3 \%$ dos pacientes. A reabsorção cervical ocorre 3 a 4 anos após a realização do enxerto ósseo secundário ${ }^{16}$.

Os EOA podem ser: autógeno, quando removidos do próprio indivíduo; homólogo, quando doado por indivíduo da mesma espécie; heterógeno, quando retirado de um indivíduo de outra espécie e aloplástico ou implante, quando produzido sinteticamente ${ }^{23}$. Dentre os biomateriais disponíveis, o osso autógeno é tido como primeira opção para a reconstrução óssea $^{2,3,11}$. O uso de enxertos ósseos removidos da crista ilíaca tem apresentado altos índices de sucesso e uma baixa incidência de complicações ${ }^{24,25}$.

Os enxertos autógenos têm potencial para reunir todos os principais fatores positivos de incorporação: osteocondução, osteoindução, osteogênese e ausência de reação imunológica ${ }^{8,26}$. Dentre as vantagens do osso autógeno destacam-se a biocompatibilidade, a presença de células osteogênicas e a osseoindução ${ }^{8,26,27}$. Por outro lado, uma desvantagem seria o seu conhecido potencial de reabsorção que pode variar entre 24 a $51 \%$ após o primeiro ano 28,29 . De acordo com um recente estudo que avaliou os preditores associados às complicações nas cirurgias de enxerto, a necessidade de a cirurgia ser refeita por insucesso foi associada a complicações no pós-cirúrgico, à idade do paciente, ao sexo e ao tipo da fissura ${ }^{30}$. A reoperação também resultou na formação de mais tecido cicatricial e futuramente poderia comprometer a irrigação sanguínea local e a cicatrização de tecidos moles e du$\operatorname{ros}^{31,32}$.

A molécula rhBMP-2 é uma das alter-
PESSOA EAM

BRAUNE A

CASADO PL

TANNURE PN

ENXERTOS ÓSSEOS

ALVEOLARES

NA FISSURA

LABIOPALATINA :

PROTOCOLOS

ATUAIS E

PERSPECTIVAS

FUTURAS

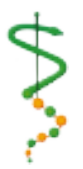

REV. ODONTOL.

UNIV. CID. SÃO

PAULO

2015; 27(1): 49 .

55, JAN-ABR 
PESSOA EAM

BRAUNE A

CASADO PL

TANNURE PN

ENXERTOS ÓSSEOS

ALVEOLARES

NA FISSURA

$\angle A B I O P A L A T I N A$ :

PROTOCOLOS

ATUAIS E

PERSPECTIVAS

FUTURAS

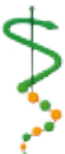

REV, ODONTOL.

UNIV, CID, SÃO

PAULO

$2015 ; 27(1): 49$

55, JAN-ABR nativas para se evitar o uso do osso da crista ilíaca ${ }^{33}$. Considerada uma proteína morfogenética óssea recombinante humana, é capaz de induzir a formação óssea quando colocada em meio adequado e age concentrando as células mesenquimais hospedeiras no local, influenciando sua diferenciação em osteoblastos ${ }^{34}$. Têm-se realizado no HRAC-USP cirurgias com rhBMP-2 e os resultados têm sido tão bons quanto os enxertos da crista ilíaca e objetiva-se eliminar a morbidade cirúrgica e a dependência de outros profissionais da área médica, fazendo com que o tratamento se torne mais simples e acessível ${ }^{10}$. O uso do rhBMP-2 tem como vantagens a recuperação no pós-operatório, assim como eliminar a necessidade de área doadora e de uma segunda equipe cirúrgica, entretanto, traz consigo um grande desafio em sua aplicação nos serviços públicos de saúde, em razão de seu custo ser extremamente elevado ${ }^{10}$.

\section{I S CUSSÃO}

Para alcançar o sucesso do tratamento com EOA torna-se indispensável coordenar a época do enxerto ósseo-alveolar da fissura com o movimento ortodôntico. A reconstrução alveolar com enxerto ósseo antes da erupção do canino permanente resulta no fechamento do defeito ósseo, obtendo-se osso favorável à finalização dos movimentos ortodônticos, melhorias na estética e total reabilitação do paciente. A erupção do canino permanente promove um estímulo intrínseco no osso enxertado, tornando esse fator fundamental na prevenção de reabsorção mantendo, assim, o nível ósseo durante a cicatrização ${ }^{35}$.

Pacientes portadores de fissuras bilaterais têm significativamente mais chance de apresentar complicações nas cirurgias de enxertos. Portadores de fissuras transforames (fissura labial e palatina) estiveram mais associados à necessidade de refazer a cirurgia, além de uma tendência a maiores complicações que os portadores de pré-forames ${ }^{30}$. Enxertos terciários também não têm apresentado os mesmos índices de sucesso dos enxertos secundários, e normalmente acarretam o comprometimento psicológico desses pacientes que são, na maioria das vezes, negligencia$\operatorname{dos}^{20,35}$.

A retirada do enxerto do osso ilíaco tem sido motivo de controvérsias devido às complicações pós-operatórias que podem ocorrer com o paciente, tais como: dor persistente, hemorragia, cicatriz visível, tempo demorado de recuperação, lesão do nervo cutâneo femoral, fratura pélvica e peritonite ${ }^{6,36,37}$. Em crianças, devido à dificuldade de se remover osso da crista ilíaca e causar uma alteração no crescimento, pode-se optar pelo enxerto costo-condral, apesar de alguns autores alegarem dificuldade de movimentação dentária nesse tipo de enxerto. Essa dificuldade ocorre devido ao componente cortical, sendo o osso esponjoso mais indicado para permitir a erupção dentária. Novas tecnologias, como a BMP, estão sendo utilizadas com objetivo de encontrar uma alternativa para o uso do osso autógeno ${ }^{33}$, entretanto, nenhuma nova técnica pode ser considerada superior ao enxerto ósseo tradicional retirado da crista ilíaca ${ }^{24}$. Além disso, mais estudos de segurança com relação ao potencial oncogenético são necessários, principalmente para uso na infância. Vale ressaltar que o uso da BMP em crianças é vetado pelo FDA (Food and Drug Adminstration) nos Estados Unidos.

É imperativa a necessidade que os pacientes portadores dessa condição têm de serem avaliados e acompanhados em centros de referência por uma equipe multidisciplinar, composta principalmente por cirurgiões, geneticistas, otorrinolaringologistas, fonoaudiólogos, cirurgiões-dentistas, entre outros. Dessa forma, desde o nascimento até a vida adulta, o suporte profissional completo estará à disposição, auxiliando o tratamento do paciente fissurado com o intuito de que, ao seu término, o estigma seja superado possibilitando, a esses pacientes, melhor inserção na sociedade $^{38}$.

Vale ressaltar que o Brasil possui alguns dos maiores centros especializados para tratamento de fissuras orais e anomalias craniofaciais, como o hospital de Reabilitação de Anomalias Craniofaciais (HRAC), ou "Centrinho", em Bauru, São Paulo e o Centro de Atendimen- 
to Integrado ao Fissurado Lábio Palatal (CAIF) em Curitiba, Paraná, dentre outros. Entretanto, mesmo com os diferentes protocolos de tratamento para os pacientes com fissuras labiopalatinas, nota-se que, em todos eles, o tratamento multidisciplinar é fundamental por buscar solucionar as necessidades estéticas e funcionais do aparelho estomatognático, bem como integrar o paciente na sociedade e proporcionar saúde psicossocial ${ }^{39}$. Diante do tema aqui discutido, vale destacar que a interação da equipe interdisciplinar com a perfeita execução das primeiras cirurgias e o correto acompanhamento ortopédico e ortodôntico são fundamentais para que a enxertia óssea seja feita no tempo correto, ou seja, próximo à erupção dentária e com as arcadas alinhadas e niveladas.

\section{CONCLUSÃO}

O defeito ósseo no rebordo alveolar é uma característica presente na maioria dos portadores de fissura labiopalatina. O enxerto ósseo alveolar é um procedimento fundamental e com evidências significativas em relação ao ganho estético e reabilitador desses pacientes, entretanto, controvérsias ainda permanecem em relação à técnica cirúrgica, ao momento da cirurgia, ao sítio de doação e se o uso de materiais alógenos apresenta melhores resultados. Diante dos fatos, pesquisas são necessárias com o objetivo de produzirem evidências fortes o suficiente para se estabelecerem protocolos únicos e otimizar o tratamento e a reabilitação dessa população.

\section{REFERÊNCIAS}

1. Mossey PA, Little J, Munger RG, Dixon MJ, Shaw WC. Cleft lip and palate. Lancet 2009 Nov 21;374(9703):177385.

2. Wehby GL. Advancing and prioritizing research on oral clefts in Brazil. J Pediatr (Rio J) 2013 Mar-Apr;89(2):112-5.

3. Jugessur A, Farlie PG, Kilpatrick N. The genetics of isolated orofacial clefts: from genotypes to subphenotypes. Oral Dis 2009 Oct;15(7):437-53.

4. Tannure PN, Soares FM, Kuchler EC, Motta LG, Costa MC, Granjeiro JM. Measuring the impact of quality of life of children treated for orofacial clefts: a case-control study. J Clin Pediatr Dent 2013 Summer;37(4):381-4.

5. Precious DS. A new reliable method for alveolar bone grafting at about 6 years of age. J Oral Maxillofac Surg 2009 Oct;67(10):2045-53.

6. Rawashdeh MA. Morbidity of iliac crest donor site following open bone harvesting in cleft lip and palate patients. Int J Oral Maxillofac Surg 2008 Mar;37(3):223-7.
7. So LL, Lui WW. Alternative donor site for alveolar bone grafting in adults with cleft lip and palate. Angle Orthod 1996 66(1):9-16.

8. Tanaka R, Yamazaki JS, Sendyk WR, Teixeira VP, França CM. Incorporação de enxerto ósseo em bloco: processo biológico e considerações relevantes. ConScientiae Saúde 2008 7(3):323-7.

9. Le BT, Woo I. Alveolar cleft repair in adults using guided bone regeneration with mineralized allograft for dental implant site development: a report of 2 cases. J Oral Maxillofac Surg 2009 Aug;67(8):1716-22.

10. Palone MRT, Silva TR, Vieira NA, Dalben GS. A importância do controle da microbiota bucal e o uso de biomaterial em cirurgias de enxerto alveolar secundário nos pacientes com fissura labiopalatina. Investigação 2013 13(2):19-23.

11. Letra A, Menezes R, Granjeiro JM, Vieira AR. AXIN2 and CDH1 polymorphisms, tooth agenesis, and oral clefts. Birth Defects Res A Clin Mol Teratol 2009 Feb;85(2):169-73.
PESSOA EAM

BRAUNE A

CASADO PL

TANNURE PN

ENXERTOS ÓSSEOS

ALVEOLARES

NA FISSURA

LABIOPALATINA :

PROTOCOLOS

ATUAIS E

PERSPECTIVAS

FUTURAS

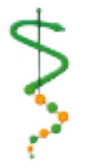

REV, ODONTOL.

UNIV. CID. SÃo

PAULO

2015; 27(1): 49 -

55 , JAN-ABR 
PESSOA EAM

BRAUNE A

CASADO PL

TANNURE PN

ENXERTOS ÓSSEOS

ALVEOLARES

NA FISSURA

LABIOPALATINA:

PROTOCOLOS

ATUAIS E

PERSPECTIVAS

FUTURAS

54

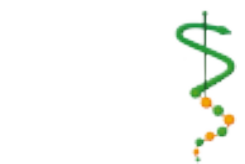

REV, ODONTOL

UNIV, CID, SÃO

PAULO

$2015 ; 27(1): 49-$

55, JAN-ABR
I SSN 1983-5183

12. Letra A, Silva RM, Motta LG, Blanton $\mathrm{SH}$, Hecht JT, Granjeirol JM, et al. Association of MMP3 and TIMP2 promoter polymorphisms with nonsyndromic oral clefts. Birth Defects Res A Clin Mol Teratol 2012 Jul;94(7):540-8.

13. Tannure PN, Oliveira CA, Maia LC, Vieira AR, Granjeiro JM, Costa Mde C. Prevalence of dental anomalies in nonsyndromic individuals with cleft lip and palate: a systematic review and meta-analysis. Cleft Palate Craniofac J 2012 Mar;49(2):194-200.

14. Kuchler EC, Saboia TM, Vieira TC, Lips A, Tannure PN, Deeley K, et al. Studies of genes involved in craniofacial development and tumorigenesis: FGF3 contributes to isolated oral clefts and may interact with PAX9. Acta Odontol Scand 2014 Nov;72(8):1070-8.

15. Saboia TM, Reis MF, Martins AM, Romanos HF, Tannure PN, Granjeiro JM, et al. DLX1 and MMP3 contribute to oral clefts with and without positive family history of cancer. Arch Oral Biol 2015 Feb;60(2):223-8.

16. Silva Filho OG, Ozawa TO, Carvalho RM. Enxerto ósseo secundário. In: Fissuras labiopalatinas: uma abordagem interdisciplinar. São Paulo: Santos; 2007. p. 337.

17. Hosokawa R, Nonaka K, Morifuji M, Shum L, Ohishi M. TGF-beta 3 decreases type I collagen and scarring after labioplasty. J Dent Res 2003 Jul;82(7):558-64.

18. Ziak P, Fedeles J, Jr., Fekiacova D, Hulin I, Jr., Fedeles J. Timing of primary lip repair in cleft patients according to surgical treatment protocol. Bratisl Lek Listy 2010 111(3):160-2.

19. Andersson EM, Sandvik L, Semb G, Abyholm F. Palatal fistulas after primary repair of clefts of the secondary palate. Scand I Plast Reconstr Surg Hand Surg 2008 42(6):296-9.
20. Dempf R, Teltzrow T, Kramer FJ, Hausamen JE. Alveolar bone grafting in patients with complete clefts: a comparative study between secondary and tertiary bone grafting. Cleft Palate Craniofac J 2002 Jan;39(1):18-25.

21. Horswell BB, Henderson JM. Secondary osteoplasty of the alveolar cleft defect. J Oral Maxillofac Surg 2003 Sep;61(9):1082-90.

22. Takahashi T, Inai T, Kochi S, Fukuda M, Yamaguchi T, Matsui K, et al. Long-term follow-up of dental implants placed in a grafted alveolar cleft: evaluation of alveolar bone height. Oral Surg Oral Med Oral Pathol Oral Radiol Endod 2008 Mar;105(3):297-302.

23. Hallman M, Thor A. Bone substitutes and growth factors as an alternative/ complement to autogenous bone for grafting in implant dentistry. Periodontol 20002008 47(172-92.

24. Guo J, Li C, Zhang Q, Wu G, Deacon SA, Chen J, et al. Secondary bone grafting for alveolar cleft in children with cleft lip or cleft lip and palate. Cochrane Database Syst Rev 2011 6):CD008050.

25. Luque-Martin E, Tobella-Camps ML, Rivera-Baro A. Alveolar graft in the cleft lip and palate patient: review of 104 cases. Med Oral Patol Oral Cir Bucal 2014 Sep;19(5):e531-7.

26. da Silva Filho OG, Boiani E, de Oliveira Cavassan A, Santamaria M, Jr. Rapid maxillary expansion after secondary alveolar bone grafting in patients with alveolar cleft. Cleft Palate Craniofac J 2009 May;46(3):331-8.

27. Borstlap WA, Heidbuchel KL, Freihofer HP, Kuijpers-Jagtman AM. Early secondary bone grafting of alveolar cleft defects. A comparison between chin and rib grafts. J Craniomaxillofac Surg 1990 Jul;1 8(5):201-5.

28. Feichtinger $M$, Mossbock R, Karcher $\mathrm{H}$. Assessment of bone resorption after secondary alveolar bone grafting using three-dimensional computed tomography: a three-year study. Cleft Palate Craniofac J 2007 Mar;44(2):142-8. 
29. Thuaksuban N, Nuntanaranont T, Pripatnanont P. A comparison of autogenous bone graft combined with deproteinized bovine bone and autogenous bone graft alone for treatment of alveolar cleft. Int J Oral Maxillofac Surg 2010 Dec;39(12):1175-80.

30. Borba AM, Borges AH, da Silva CS, Brozoski MA, Naclerio-Homem Mda G, Miloro M. Predictors of complication for alveolar cleft bone graft. $\mathrm{Br} / \mathrm{Oral}$ Maxillofac Surg 2014 Feb;52(2):1748.

31. Milstein DM, Cheung YW, Ziukaite L, Ince $C$, van den Akker HP, Lindeboom JA. An integrative approach for comparing microcirculation between normal and alveolar cleft gingiva in children scheduled for secondary bone grafting procedures. Oral Surg Oral Med Oral Pathol Oral Radiol 2013 Mar;115(3):304-9.

32. Poenaru D. Getting the job done: analysis of the impact and effectiveness of the SmileTrain program in alleviating the global burden of cleft disease. World J Surg 2013 Jul;37(7):1562-70.

33. Coots BK. Alveolar bone grafting: past, present, and new horizons. Semin Plast Surg 2012 26(4):178-83.

34. Chen D, Zhao M, Mundy GR. Bone morphogenetic proteins. Growth Factors 2004 Dec;22(4):233-41.
35. Trindade-Suedam IK, da Silva Filho OG, Carvalho RM, de Souza Faco RA, Calvo AM, Ozawa TO, et al. Timing of alveolar bone grafting determines different outcomes in patients with unilateral cleft palate. J Craniofac Surg 2012 Sep;23(5):1283-6.

36. Dawson KH, Egbert MA, Myall RW. Pain following iliac crest bone grafting of alveolar clefts. J Craniomaxillofac Surg 1996 Jun;24(3):151-4.

37. Sbitany H, Koltz PF, Amalfi AN, Waldman J, Girotto JA. Pain control in the anterior iliac crest following bone graft harvest. Plast Reconstr Surg 2010 May;125(5):215e-6e.

38. Alonso N, Tanikawa DYS, De Lima Junior JE, Rocha DL, Sterman S, Ferreira MC. Fissuras labiopalatinas: protocolo de atendimento multidisciplinar e seguimento longitudinal em 91 pacientes consecutivos. Rev bras cir plást 2009 abr-jun;24(2):176-81.

39. Antunes CL, Aranha AMF, Lima E, Pedro FLM, Shimoya-Bittencourt W, Pereira ICL, et al. Planejamento ortodôntico para pacientes portadores de fissuras labiopalatinas: revisão de literatura. UNOPAR Cient Ciênc Biol Saúde 2014 16(3):239-43.

Recebido em 08/06/2015

Aceito em 15/06/2015
PESSOA EAM

BRAUNE A

CASADO PL

TANNURE PN

ENXERTOS ÓSSEOS

ALVEOLARES

NA FISSURA

LABIOPALATINA:

PROTOCOLOS

ATUAIS E

PERSPECTIVAS

FUTURAS

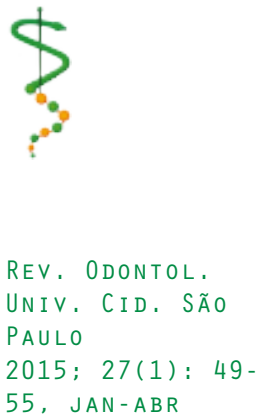

